\title{
P. Valéry, S. De Madariaga, Correspon-dance pour une Société des Esprits
}

\section{Paola Cattani}

\section{(2) OpenEdition}

1 Journals

\section{Édition électronique}

URL : http://journals.openedition.org/studifrancesi/10587

DOI : 10.4000/studifrancesi. 10587

ISSN : 2421-5856

Éditeur

Rosenberg \& Sellier

\section{Édition imprimée}

Date de publication : 1 décembre 2017

Pagination : 580-581

ISSN : 0039-2944

\section{Référence électronique}

Paola Cattani, «P. Valéry, S. De Madariaga, Correspon-dance pour une Société des Esprits », Studi Francesi [En ligne], 183 (LXI | III) | 2017, mis en ligne le 01 février 2018, consulté le 23 janvier 2021. URL : http://journals.openedition.org/studifrancesi/10587 ; DOI : https://doi.org/10.4000/ studifrancesi. 10587

Ce document a été généré automatiquement le 23 janvier 2021.

\section{(c) 9 (i) $\Theta$}

Studi Francesi è distribuita con Licenza Creative Commons Attribuzione - Non commerciale - Non opere derivate 4.0 Internazionale. 


\title{
P. Valéry, S. De Madariaga, Correspon-dance pour une Société des Esprits
}

\author{
Paola Cattani
}

\section{RÉFÉRENCE}

PAUL VALÉRY, SALVADOR DE MADARIAGA, Correspon-dance pour une Société des Esprits, préface de Jean-Michel Rey, Università di Corsica, Éditions Albiana, 2016, «Estru Mediterraniu», $67 \mathrm{pp}$.

1 Après 1925, la Société des Nations multiplie les initiatives vouées à favoriser la coopération intellectuelle entre les hommes de lettres européens. L'Institut international de coopération intellectuelle, créé dans ce cadre en 1926 et ayant siège à Paris, met en place un riche programme de conférences et de publications, auxquelles prennent part entre autres Paul Valéry, Thomas Mann, Johan Huizinga, Julien Benda, Aldous Huxley, œuvrant tous en faveur de la paix et de l'unité d'une Europe de plus en plus menacée par les nationalismes et les totalitarismes. Parmi les initiatives les plus significatives figurent la série des huit «Entretiens», des conférences internationales consacrées à l'avenir de l'esprit et de la culture européens, et la publication de correspondances publiques d'écrivains sur des questions d'actualité. Il s'agit d'un chantier très riche, non seulement pour les personnalités qui s'y investissent, mais aussi pour l'intérêt des échanges portant sur la crise du monde moderne et sur la fonction de la culture et de l'homme de lettres.

2 Les éditions Albiana viennent de rééditer en partie l'une des correspondances publiées en 1933 par la Société des Nations: les lettres de Paul Valéry et de l'écrivain, historien et diplomate espagnol Salvador De Madariaga (1886-1978) portant sur la «Société des esprits» (la publication originaire comprenait aussi des lettres de Alfonso Reyes, Miguel Ozorio de Almeida, Gilbert Murray, Tsaï Yuan Peï). Ce volume a donc avant tout le 
mérite d'attirer l'attention sur des documents peu connus et fréquentés, même par la critique intéressée au débat littéraire de l'entre-deux-guerres. Aussi et surtout, il fournit l'occasion de repenser les modalités et les présupposés de la "politique de l'impossible» à laquelle se voue la République des lettres de l'entre-deux-guerres, et à laquelle est consacré l'essai-préface de Jean-Michel Rey qui ouvre le volume.

3 Au cœur de la Correspondance gît l'idée d'une «Société des Esprits» appelée à conduire l'Europe dans les troubles qui la secouent après le premier conflit mondial. Il s'agit d'une notion créée par Valéry et qui a fait sa première apparition dans le rapport écrit par celui-ci en juillet 1930 pour la Commission internationale de coopération intellectuelle, intitulé Société des Nations et Société des Esprits; elle a ensuite été mise au grand jour précisément par cette correspondance publique de 1933. Un telle «Société» attribue une place privilégiée dans le monde à l'«esprit» invoqué (avec ou sans majuscule) de toutes parts dans l'entre-deux-guerres. Romain Rolland, par exemple, publie dans l'immédiat après-guerre ses deux manifestes Pour l'internationale de l'Esprit et Déclaration d'indépendance de l'Esprit, plaidant en faveur d'un "pan humanisme» universel et d'un «internationalisme de la culture»; d'autre part, quelques années plus tard, André Breton réclamera la nécessité d'une «révolte de l'Esprit» pour parvenir à la «dictature de l'Esprit», puisque ce n'est à ses yeux qu'à travers la révolte et la révolution que l'art pourra réaffirmer son pouvoir. À la différence de Rolland, Valéry ne met pas en avant la nécessité de sauvegarder l'art des influences extérieures, mais le thème de sa supériorité sur le monde; à la différence des surréalistes, il ne fonde pas cette suprématie sur une action subversive telle que la révolte ou la révolution, mais sur une raison de droit.

4 Le volume qui vient d'être réédité permet de situer la notion et les propos de Valéry par rapport à leur contexte originaire. La lettre que Valéry adresse à De Madariaga dans la correspondance publique, se trouve aussi reproduite dans Variété, où elle entre en résonance avec les autres «Essais quasi-politiques» de Valéry, et notamment avec le texte de la conférence «La politique de l'esprit», donnée en 1932 à l'Université des Annales. Mais la signification et les enjeux de la formule valéryenne se précisent avant tout dans le cadre des échanges développés au sein des organismes de coopération intellectuelle de la Société des Nations, à savoir la correspondance publique ainsi que les deux Entretiens organisés en ce même 1933 sur «L'Avenir de la Culture» et "L'Avenir de l'Esprit européen», revenant sur le même questionnement qui se trouve au cœur de la Correspondance: «Que devient l'homme de pensée, si l'ordre intellectuel n'est pas fortement défini, s'il n'est pas établi que, par delà l'animalité des instincts, par delà les intérêts des classes, des partis, des nations, résident des intérêts supérieurs, dont l'ordre intellectuel a la charge?» (p. 28).

5 Comme Rey le signale dans sa préface, l'Esprit tel que Valéry et ses interlocuteurs le conçoivent est une véritable «puissance de transformation». La réalité ne saurait en effet exister, aux yeux de Valéry, sans «les rêves et les mythes» qui l'appuient et l'orientent. L'Esprit joue également un rôle crucial en tant que puissance de résistance, ne se pliant pas à la nécessité de conclure ou d'agir. Ainsi, sur un plan strictement politique, la Société des Nations ne peut trouver son crédit et sa mission sans une Société des Esprits préalable, qui accompagne et transcende en même temps la réalité de la politique, des partis, des nations.

6 Considéré à partir d'un telle perspective, le reproche que Sartre, Benda et d'autres adresseront au lendemain de la Seconde Guerre mondiale aux hommes de lettres de 
l'entre-deux-guerres, leur attribuant une part de responsabilité intellectuelle par rapport au désastre survenu, est tout à fait infondé. L'Esprit tel qu'il est conçu par Valéry et par ses interlocuteurs est en effet l'autre du Monde, avec lequel il entretient un rapport dialectique, un commerce et un va-et-vient incessants et salvifiques qui essaient de faire dialoguer Possible et Impossible. "Que serions-nous donc sans le secours de ce qui n'existe pas? Peu de chose, et nos esprits bien inoccupés languiraient si les fables, les méprises, les abstractions, les croyances et les monstres, les hypothèses et les prétendus problèmes de la métaphysique ne peuplaient d'êtres et d'images sans objets nos profondeurs et nos ténèbres naturelles» (Petite lettre sur les mythes, 1928).

7 Par ailleurs, le volume de la Correspondance témoigne aussi de l'existence concrète et effective de la République des Lettres des débuts du $\mathrm{xx}^{\mathrm{e}}$ siècle. En choisissant la forme de la correspondance publique, ces auteurs revivifient d'une part le moyen de communication et d'échange privilégié de la République des Lettres des $\mathrm{XVI}^{\mathrm{e}}-\mathrm{XVII}{ }^{\mathrm{e}}$ siècles, dont ils réaffirment, face aux troubles du siècle nouveau, le modèle et l'idéal. D'autre part, ils se dressent en défense de l'échange et du dialogue en tant que modalités communicatives qui s'accompagnent d'une attitude éthique: il s'agit pour eux d'accomplir à la fois un acte de partage, qui essaie de surmonter l'individualité de l'écriture, et un acte d'humilité et d'ouverture véritable, qui se refuse à la polémique en tant que modalité discursive conflictuelle. C'est donc par le biais de la lettre en tant que moyen de "dialogue», que ces hommes de lettres essaient d'aborder, et de replacer au centre du débat public et politique, la question de «Que veut-on faire de l'homme, et quel homme veut-on faire?» (p. 26). 